\title{
A study of IRFPA camera measurement errors: radiometric artefacts
}

\author{
Martin Poncelet ${ }^{\dagger}$ - Jean-François Witz ${ }^{*}{ }^{\dagger}-$ Hervé Pron ${ }^{\ddagger}-$ \\ Bertand Wattrisse ${ }^{\S}$
}

"now at: LML UMR 8107 - 59655 Villeneuve d'Ascq cedex, France

jean-francois.witz@ec-lille.fr

${ }^{\dagger}$ LMT-Cachan (ENS Cachan/CNRS/Université Paris 6/PRES UniverSud Paris)

61 avenue du Président Wilson, F-94235 Cachan Cedex, France

${ }^{\dagger}$ Université de Reims / GRESPI - EA 4301, Faculté des Sciences - CC n²1

Campus du Moulin de la Housse, BP 1039, F-51687 Reims CEDEX 2, France

herve.pron@univ-reims.fr

${ }^{\S}$ LMGC UMR-CNRS 5508, Université Montpellier II

Place E. Bataillon 34095 Montpellier Cedex 5, France

wattriss@lmgc.univ-montp2.fr

\begin{abstract}
Infrared Focal Plane Array cameras have several aberrations and measurement artefacts with different material origins: lenses, sensors, Read-Out-Circuits of the sensors. The authors' goal is to characterize each artefact separately to obtain more versatile correction procedures and improve the correction accuracy. This paper focuses on some radiometric artefacts associated with variations of measured illuminance. It presents a new correction algorithm dedicated to Narcissus and vignetting effects, which is identified and validated for two different lenses. It also proposes an innovative experimental protocol to detect low-level contrast-induced artefacts. Several $R \& D$ cameras and lenses are tested and some present such artefacts, which can not be corrected by traditional pixel-to-pixel methods. Their origins are shown to derive either from the optical system or the Read-Out-Circuit.

KEY WORDS: IRFPA camera, artefacts, aberrations, non uniformity correction, IR optics, vignetting.
\end{abstract}

Received October 23, 2010; accepted for publication February 11. 


\section{Introduction}

The use of Infrared Focal Plane Array (IRFPA) cameras for quantitative thermal field measurements is more and more widespread in the industrial and scientific communities. Even though scientific reasons justify this recent interest (e.g. (Chrysochoos et al., 1989), (La Rosa et al., 2000), (Chrysochoos 2002), (Louche et al., 2005), (Bouferra et al., 2005), (Doudard et al., 2007), (Pastor et al., 2008)), the pragmatic reasons for this involvement is the availability of affordable IRFPA cameras on the civil market and their numerical array data format that simplify data processing.

When an accurate thermal field measurement is required, one faces the complexity of such a measurement system. To tackle low-level heterogeneities (i.e less than $10 \%$ of the thermal dynamic range of the studied scene), one has to take into account the external and internal conditions of measurement. In the field of experimental mechanics for example, the thermal heterogeneities in the scene (e.g. actuators, lighting) may be several orders of magnitude above the sought ones in a particular region (e.g. heat sources related to material plasticity) (Poncelet et al., 2010), so that their influence has to be studied. These camera-related artefacts are not only due to the optical part of the camera. For instance, the conversion of photons into electrical charges by each elementary sensor, the transmission of each pixel information through the Read-OutCircuit (ROC) and the following image processing circuits may have other deleterious effects (Pron et al., 2004). These effects are considered as "radiometric artefacts" when they induce a change in the measured luminance of the object) or "geometrical aberrations" when they induce an apparent displacement or deformation of the object).

Knowing the specifications of all the components (lens, sensor, ROC, etc.) is essential to correct these artefacts. Because of the scarcity of available specifications, one has to evaluate them by oneself. Unfortunately, most of the users only have their IRFPA camera at hand and not the necessary thermal, optical and electronic devices to characterise the different components of their camera independently.

For this first reason, and because the goal of the user is, in the end, the correction of the camera and not that of its components, the usually proposed methods (e.g. (Schulz et al., 1995), (Honorat et al., 2005), (Shi et al., 2005), (Kumar et al., 2007)) consider the camera as a black box, and the corrections are in fact calibrations of the measuring chain as a whole. These methods do not distinguish between the influences of the different components of the camera. A new calibration is therefore needed after an exchange of lenses, a different lens focus, a different integration time, a different sub-windowing, etc. Moreover, such calibration methods are based on the observation of an extended black body, and thus pixels data interdependencies, only revealed by non-uniform targets, can not be corrected.

More generally, the choice of the calibration functions is very often not justified by physics, so that the calibration needs a lot of parameters to achieve enough accuracy, contrary to physics-based methods that use very few parameters, each of them having a particular meaning (Castelein 2003). The quality of these latter methods is naturally 
closely linked to the validity of the underlining models. It is thus necessary to highlight the different artefacts that can be encountered in a real measurement conditions.

The goal of this paper is to propose some alternative methods based on simple experimental protocols (i.e. they do not imply complex or expensive optical set-up) to characterise and correct some radiometric artefacts, namely the Narcissus and vignetting effects, and also pixel interdependencies that may be generated by ROC and lens. It does not consider measurement uncertainties, but systematic errors (GUM, 2008). The different "steps" of corrections are as much as possible related to the different component of the system and justified by physics laws.

In a first part, a brief description of the different origins of artefacts will be presented, underlining their specific features, and thus helping in the understanding of the experimentally obtained results. In a second part, the tested devices (two IRFPA camera bodies combined with two IR lenses) will be described. In a third part, an algorithm for the correction of vignetting and Narcissus effects will be explained and applied to the lenses. In a fourth part, an experimental protocol for the detection of contrast-induced artefacts will be detailed and applied to the different cameras and lenses. Last, a discussion on the observed artefacts and their corrections will follow.

\section{Origins and features of the artefacts}

From now on, one will make a distinction between the illuminance $E$, the measured illuminance $E^{m}$ (i.e. the equivalent electrical charges accumulated in a photodetector) and the read illuminance $E^{r}$ (i.e. the equivalent numerical value given by the camera). A brief description of the three corresponding steps of measurement, namely the image-forming, the electronic conversion and the electronic reading, will now be presented to show the possible artefacts and their main characteristics.

\subsection{Optical artefacts}

From an optical point of view, an experimental infrared measurement set-up is summed up to its three fundamental elements: the object in the object plane $(y, z)$, the IRFPA sensor in the image plane $\left(y^{\prime}, z^{\prime}\right)$ and between them the optical system with its optical axis $x$. Every parameter and variable depends on the wavelength $\lambda$ of the considered radiation. This dependence is not represented to simplify the equations. The optical relation between the luminance $L$ of the object at a given point $P(y, z)$ and its corresponding illuminance $E$ of the IRFPA sensor at the image point $P^{\prime}\left(y^{\prime}, z^{\prime}\right)$ reads to the first order

$$
E\left(y^{\prime}, z^{\prime}\right)=C L(y, z)
$$

where $C$ is the "transmission coefficient" given by the manufacturer of the lens. No matter how simple, this first relation is sufficient if one uses the set-up as an imaging system. 
When the set-up is used as a thermal field measuring system, one must consider several optical artefacts that may have deleterious effects on the measured temperature field.

- First, the so-called "transmission coefficient" is more precisely a mean transmission coefficient. A variation of the transmission coefficient $f(y, z)$ with the considered point has to be taken into account. The most well-known artefact in this category is the "vignetting" effect. It corresponds to a transmission coefficient decreasing with the distance to the optical axis, causing a darkening of the image at its periphery, where signal is only due to the temperature of the camera case. Moreover, for a majority of sensors, the more tilted the rays, the less sensible the elementary sensor. However this second artefact is not stricto-sensu linked to the lens (but to the lens-sensor combination), one includes it in the vignetting effect in this paper. This type of artefact is multiplicative.

- Second, the luminance $L_{r}$ owing to the reflection of the surrounding must be added to the luminance $L$ of the studied point $P$ due to its own temperature. A particular element of the surrounding is the camera itself, whose reflection on the object is responsible for the phenomenon called "Narcissus effect". Consequently one considers this type of artefact - at least the Narcissus effect - as "partially-internal" because it depends not only on the camera, but also on the relative position of the object in front of the camera, its shape and emissivity.

- Third, a second source placed at a different point in the object plane $P_{s}\left(y_{s}, z_{s}\right)$ may have a contribution to the illuminance $E\left(y^{\prime}, z^{\prime}\right)$ because of complex multiple reflections inside the optical system. These types of artefacts are referred to as "lens flare" in the field of photography. Some of these artefacts are due to (unwanted) reflections between the optical system and the object, hence showing some similarity with the Narcissus effect. These artefacts are to be added to the previous ones.

Consequently a more realistic form of the Eq. (1) reads:

$$
E\left(y^{\prime}, z^{\prime}\right)=\underbrace{f(y, z)}_{\text {Vignetting }}(\underbrace{L(y, z)}_{\text {Object luminance }}+\underbrace{L \cdot(y, z)}_{\text {Narcissus }})+\underbrace{\iint_{y_{r}, z r} g\left(y, z, y_{r}, z_{r}\right) L\left(y_{r}, z_{r}\right) d y_{r} d z r}_{\text {"Lens flare" }},
$$

where $g$ stands for the correlation between points $P$ and $P_{s}$ due to multiple reflections.

The study of geometrical aberrations, such as the barrel or pincushion distortions, will not be in the scope of this paper. However, one must keep in mind that they induce an illumination measurement error (as long as the measured field is heterogeneous) through the introduction of a displacement of the image point.

\subsection{Sensor artefacts}

They come from the heterogeneity of sensibility of the elementary photodetectors and from the diffusion of charges in the sensor. An independent calibration of each pixel, as proposed by (Honorat et al. 2005), corrects exclusively heterogeneity 
problems but only if performed on a uniform black body with a collimator. On the contrary, the interdependency between close pixels is to be considered to correct diffusion effects, for example by writing to the first order the measured illuminance $E^{m}$ at the $(i, j)^{\text {th }}$ pixel as follows

$$
E_{i j}^{m}=S_{i j}\left(E\left(y_{i}, z_{j}\right)\right)+\sum_{k, l} B_{k-i, l-j}\left(S_{k l}\left(E\left(y_{k}, z_{l}\right)\right)-S_{i j}\left(E\left(y_{i}, z_{j}\right)\right)\right)
$$

where $\left(y_{i}, z_{j}\right)$ are the geometrical coordinates of the $(i, j)^{\text {th }}$ pixel. $S_{i j}$ is the calibration function of the $(i, j)^{\text {th }}$ pixel (a polynomial function in most cases), whose dependence with the working temperature of the sensor is neglected since the camera are used in steady-state conditions in this study. The matrix $B$ links the difference of illuminance between the considered pixel and its neighbours to the measured illuminance. For a diffusion-free sensor, one has $B=0$.

\subsection{Read-Out-Circuit artefacts}

The Read-Out-Circuit may generate interactions between pixels information during the sequential reading. Depending on the ROC, the reading order is not necessarily Figure 1a), but may be far more complex to enable various sub-windowing possibilities. An example of such an order is given Figure 1b, corresponding to the Cedip Jade III Camera. A general expression of the read illuminance $E^{r}$ is written

$$
E_{i j}^{r}=E_{i j}^{m}+\sum_{n} R_{i j n}\left(E_{I_{n} J_{n}}^{m}-E_{i j}^{m}\right)
$$

where $n$ ranges from 0 (the current pixel) to $\mathrm{N}$, the last read pixel whose influence on the current one is detectable. The indices $I_{n}$ and $J_{n}$ describe the reading order as illustrated in Figure 1 and the coefficient $R_{i j n}$ the attenuation of the last but $n$ read pixel on the current pixel $(i, j)$.

From this brief overview of the different origins and features of potential artefacts, one sees that the distinction between each of them may be difficult when the camera components are not tested separately. Nonetheless, these artefacts may be distinguished through several considerations. First and foremost, the features of an artefact are linked to its origins. For instance, optical ones can reasonably be supposed to be symmetrical with respect to the optical axis. Diffusion ones can be supposed to be identical from one pixel to another and to be localised in the neighbourhood of the given pixel. Finally ROC-related ones should induce particular patterns between rows and columns, etc. Second, exchanging camera components when possible (lenses for example) enables to deduce which artefacts is or is not due to the component. Last, changing the settings (focus, integration time, sub-windowing, etc.) helps understand the origins of artefacts. The next section will present the devices whose radiometric 
artefacts will be searched for, namely the vignetting and Narcissus effects and the contrast-induced artefacts, which combine "lens flare" and ROC-related artefacts.

\begin{tabular}{|c|c|c|c|c|c|}
\hline 1 & 2 & 3 & 4 & 5 & 6 \\
\hline 7 & 8 & 9 & 10 & 11 & 12 \\
\hline 13 & 14 & 15 & 16 & 17 & 18 \\
\hline 19 & 20 & 21 & 22 & 23 & 24 \\
\hline
\end{tabular}

\begin{tabular}{|c|c|c|c|c|c|}
\hline 1 & 5 & 9 & 2 & 6 & 10 \\
\hline 13 & 17 & 21 & 14 & 18 & 22 \\
\hline 15 & 19 & 23 & 16 & 20 & 24 \\
\hline 3 & 7 & 11 & 4 & 8 & 12 \\
\hline
\end{tabular}

Figure 1. Two different reading orders: (a) standard top-left-to-bottom-right scanning (b) 4-channels multiplexed reading as used in the Cedip Jade III camera.

\section{Tested devices}

The two investigated IRFPA cameras are presented in Table 1. Both are produced by CEDIP-FLIR and are based on a Stirling-cooled InSb sensor allowing a high sensibility in middle wave infrared wavelength band. Even if it is not mentioned by the manufacturer, one assumes two totally different types of ROC from the differences of resolution and sub-windowing possibilities.

\begin{tabular}{|l|c|c|}
\hline & Jade III & Titanium SC 7600 \\
\hline Type of sensor & InSb & InSb \\
\hline Pitch & $30 \mu \mathrm{m}$ & $15 \mu \mathrm{m}$ \\
\hline Spatial resolution & $320 \times 240$ pixels & $640 \times 512$ pixels \\
\hline Max. frequency in full frame mode & $150 \mathrm{fps}$ & $100 \mathrm{fps}$ \\
\hline Spectral bandwith & $3.5-5 \mu \mathrm{m}$ & $1.5-5.1 \mu \mathrm{m}$ \\
\hline NETD given by the maker & $20 \mathrm{mK}$ at $20^{\circ} \mathrm{C}$ & $20 \mathrm{mK}$ at $20^{\circ} \mathrm{C}$ \\
\hline Temperature of the detector & $70 \mathrm{~K}$ & $70 \mathrm{~K}$ \\
\hline Sub-windowing & $\begin{array}{c}1 / 2 \text { frame (centered) } \\
1 / 4 \text { frame (centered) } \\
64 \times 8 \text { (centered) }\end{array}$ & $\begin{array}{c}\text { Free size and } \\
\text { free position } \\
(64 \times 4 \text { at minimum) }\end{array}$ \\
\hline
\end{tabular}

Table 1. Main characteristics of the two IRFPA cameras. 
Table 2 presents the chosen lenses: a $50 \mathrm{~mm}$ prime lens and a microscope lens. Both are designed by CEDIP-FLIR to be used with either camera, yet the bandwith of the MW x1 lens is thinner than the bandwith of the Titanium camera. The microscope lens has worse features in terms of transmission and distortion (CEDIP 2004, CEDIP 2005) but allows a high magnification (x1). The manufacturer of the prime lens specifies the absence of vignetting when focus is higher than $1500 \mathrm{~mm}$. In this paper, the prime lens is always used near the minimum working distance (about $300 \mathrm{~mm}$ ).

In the case of the vignetting effect, the study will be limited to the Jade camera equipped with the two lenses. On the contrary, investigations of the contrast-induced artefacts will be performed on the 4 possible setups combinations to deduce and characterise the different artefacts of each component. Each study requires a planar extended differential black body. DCN1000 black body produced by HGH will be used. Its active surface is $100 \times 100 \mathrm{~mm}^{2}$, the given emissivity in the middle wave wavelength band is higher than $0.97 \%$, the specified spatial non-uniformity is \pm $0.010 \mathrm{~K}$ at room temperature $\pm 5 \mathrm{~K}$, and its stability is $\pm 0.002 \mathrm{~K}$.

\begin{tabular}{|l|c|c|}
\hline & MW 50 mm & MW x1 \\
\hline Focal & $50+/-0.5 \mathrm{~mm}$ & $10.45+/-0.5 \mathrm{~mm}$ \\
\hline F/number & $2+/-5 \%$ & $2+/-10 \%$ \\
\hline Horizontal Field of view & $10.97^{\circ}$ & $9.6 \mathrm{~mm}$ \\
\hline Spectral bandwith & $3.5-5+/-0.25 \mu \mathrm{m}$ & $3.5-5+/-0.25 \mu \mathrm{m}$ \\
\hline Mean transmission coefficient & $>94 \%$ & $>85 \%$ \\
\hline
\end{tabular}

Table 2. Main characteristics of the two IR lenses.

\section{Axisymmetrical radiometric artefacts detection and correction}

One focuses in this section on the axisymmetrical artefacts due to optical phenomena, namely the vignetting and Narcissus effects (in the case of a planar object normal to the optical axis).

\subsection{Experimental protocol}

The camera is placed in front of the black body. Two different black body temperatures $T_{B B 1}$ and $T_{B B 2}$ are chosen (enough for the considered integration time and under the saturation temperature). The camera is not focused on the active surface of the black body but $10 \mathrm{~mm}$ in front of it, where the target will be placed in due time. The embedded non uniformity correction of the camera is disabled. At each 
temperature a film of several hundreds of images is acquired and a time average is performed to reduce temporal noise. The unit of these images are "digital level" of temperature (DL). Using the global calibration curve of the camera $T(i, j)=f_{\text {cal }}\left(E^{r}(i, j)\right)$, one converts DL into the equivalent apparent temperature $T$ at the pixel $(i, j)$ in Kelvin $(\mathrm{K})$. This temperature is then converted into a emitted flux $\left(\mathrm{w} / \mathrm{m}^{2}\right)$ with the StefanBoltzman law $E^{r}(i, j)=\varepsilon_{B B} \sigma_{s b} T(i, j)^{4}$ where $\varepsilon_{B B}$ is the emissivity of the black body and $\sigma_{s b}$ the Stefan-Boltzman constant. Figure 2 shows the result for $T_{B B I}=25^{\circ} \mathrm{C}$ with a scale normalized by the maximum value of the image, i.e. $E^{r}(\%)=E^{r} / \max \left(E^{r}\right)$. The vignetting effect is clearly noticeable: a uniform read illuminance was expected because of the extended black body, but one observes an important decrease toward the edge of the image (about $20 \%$ ). The emissivity of the black body is high, so that the Narcissus effect is not visible because of its low magnitude.

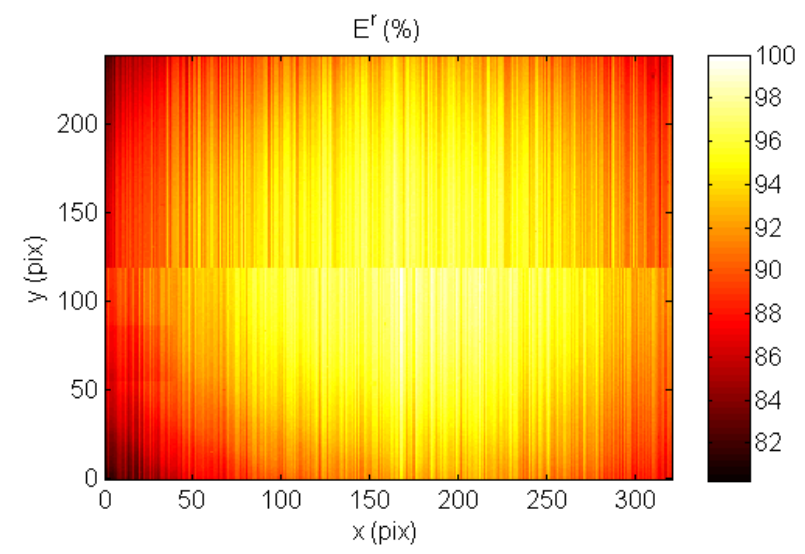

Figure 2. Non-corrected image of the black body, normalized by the maximum value of the image $\left(50 \mathrm{~mm}\right.$ prime lens, black body temperature $=25{ }^{\circ} \mathrm{C}, \mathrm{IT}=$ $1400 \mu \mathrm{s})$. The "unmatched columns" and the step between the upper and lower halves are electronic artefacts, corresponding to 4-channels multiplexed ROC.

\subsection{Correction Algorithm}

In the present case, one does not consider the second term of the right hand member of Eq. [2] that stands for "lens flare" artefact type, because of its very low relative magnitude. Consequently Eq. [2] is simplified to:

$$
E\left(y^{\prime}, z^{\prime}\right)=f(y, z)(L(y, z)+L \cdot(y, z))
$$

Because of the axisymmetry of the lens, the vignetting is modeled as a transmission function $f$ of the radius to the optical axis. A radial polynomial function 
$P$ (i.e. composed of even powers and only depending on the radius) is thus relevant to model such axisymmetrical phenomenon with very few parameters. For the same reasons, the Narcissus effect is modeled as an external luminance function $L_{r}$ that is also a radial power function. The origins of the coordinate systems used for each function are different from the sensor centre. First because most manufacturers tolerate a sensor shift with respect to the optical axis (around a few pixels). Second because the optical axis is actually not normal to the black body during experiment, which induces a difference of vignetting centre and Narcissus centre.

The transmission function $f\left(r_{v}\right)$ is fitted on the difference between the two images at $T_{B B 1}$ and $T_{B B 2}$, so that the Narcissus effect does not affect identification. The external luminance function $L_{r}\left(r^{\prime}\right)$ is then fitted on the first of the two images. In both cases, the least-square method is used. Lastly any image $E^{r}$ is corrected as follows:

$$
E^{c o r}(i, j)=\frac{E^{r}(i, j)-f(i, j) L(i, j)}{f(i, j)} .
$$

Each pixel is thus corrected linearly with an offset $-L_{r}$ and a gain $1 / f$, in the same way as the usual " 2 points Non-Uniformity-Correction". The important difference lies in the number of necessary parameters. The 2 points Non-Uniformity-Correction uses a number of parameters equal to twice the number of pixels since each pixel $(i, j)$ correction requires different coefficients $L_{r}(i, j)$ and $f(i, j)$. On the contrary the proposed correction requires less than a dozen, since the gain and offset applied to each pixel come from the same two polynomial functions $L_{r}\left(r^{\prime}\right)$ and $f\left(r_{v}\right)$.

\subsection{Application}

Two images $E_{1}^{r}$ and $E_{2}^{r}$ are taken at respectively 25 and $35^{\circ} \mathrm{C}$. An other image $E_{3}^{r}$ is recorded at $30^{\circ} \mathrm{C}$. A last image $E_{4}^{r}$ is taken with the black body at $30^{\circ} \mathrm{C}$ and a semicircular target in front of it. Figures $3 \mathrm{a}, 3 \mathrm{~b}, 3 \mathrm{c}$, and $3 \mathrm{~d}$ present them. For the sake of clarity, images are normalised on this figure by their respective range, i.e. $E^{r}(\%)=\left(E^{r}-\min \left(E^{r}\right)\right) /\left(\max \left(E^{r}\right)-\min \left(E^{r}\right)\right)$. Correction functions are fitted using images $E_{1}^{r}$ and $E_{2}^{r}$. Polynomials of the $4^{\text {th }}$ degree are used for the transmission and the external luminance function, so that the correction algorithm has only six parameters. Higher degrees allows only minor improvements. All four images are then corrected and presented Figures 3a', 3b', 3c', and 3d'. To ease comparison with initial images, they are normalized by the range of their respective non-corrected image, i.e. $E^{\operatorname{cor}}(\%)=\left(E^{c o r}-\min \left(E^{r}\right)\right) /\left(\max \left(E^{r}\right)-\min \left(E^{r}\right)\right)$.

Figures 3a' and 3b' show the residues of the identification, i.e. the images $E_{1}^{r}$ and $E_{2}^{r}$ corrected by the algorithm that is identified on these same images. No longer halos are present, only vertical and horizontal patterns are visible, which correspond to the sensor non-uniformity. The standard deviation of the uncorrected images is about $15 \%$, 
while corrected one is about $6 \%$. Even though the level of heterogeneity is still important due to this last electronic artefact, the global level is noticeably less important.

Figures 3c' and 3d' show validations of the correction algorithm, i.e. the images $E_{3}^{r}$ and $E_{4}^{r}$ corrected by the algorithm identified on the other two images $E_{1}^{r}$ and $E_{2}^{r}$. In comparison with the corresponding non-corrected images, the remaining halo is negligible. Once more the standard deviation drops from $15 \%$ to $6 \%$, most of the remaining visible heterogeneity being again due to the sensor. In Figure $3 \mathrm{~d}$ ' both the target and the black body behind it are far more uniform than in the initial image $3 \mathrm{~d}$ ).

This correction method is also applied to the microscope lens. The identification is performed in two images at $25^{\circ} \mathrm{C}$ and $35^{\circ} \mathrm{C}$. An image at $30^{\circ} \mathrm{C}$ is then corrected. When the corrected image is normalized by the range of its non-corrected image, it is not differentiable from the corresponding image obtained with the prime lens: no halo is visible, only sensor non-uniformity is noticeable. More precisely, the difference between the corrected images obtained with the prime and the microscope lens at $30^{\circ} \mathrm{C}$ is less than $1 \%$. This proves that only the electronic artefact remains after that the correction algorithm is applied.

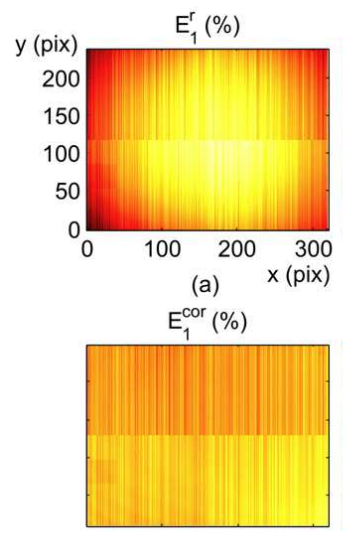

(a')

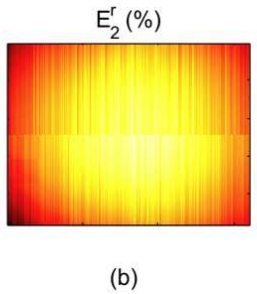

$\mathrm{E}_{2}^{\mathrm{cor}}(\%)$

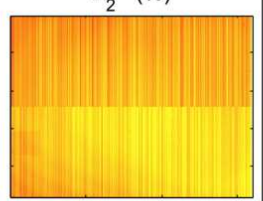

(b')

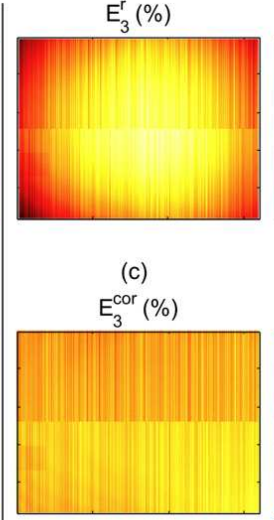

$\left(c^{\prime}\right)$

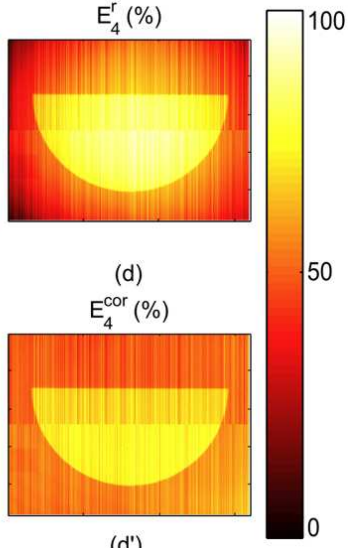

$\left(d^{\prime}\right)$

Figure 3. $(a, b)$ Raw image at $25^{\circ} \mathrm{C}$ and $35^{\circ} \mathrm{C}$ used for identification; ( $\left.a^{\prime}, b^{\prime}\right)$ corresponding residues of the identification; (c) raw image at $30^{\circ} \mathrm{C} ;(\mathrm{d})$ raw image of the semi-circular target ; $\left(c^{\prime}, d^{\prime}\right)$ corresponding corrected images $(50 \mathrm{~mm}$ prime lens, $I T=1400 \mu s)$.

\section{Contrast-induced radiometric artefacts detection}

This section is dedicated to the "contrast-induced" artefacts, i.e. to the second term of the right hand member of Eq. (2). Since these artefacts correlate the read illuminance of different points of the image, one can not detect them without black body target. 


\subsection{Experimental protocol}

A special removable target is designed to measure contrast-induced artefacts. It is an improvement compared to a target proposed before (Poncelet 2007). It generates a periodic, uniform and lambertian penny-shaped source of radiation placed on an infinite reflective plane. The advantages of such a target are:

- The size of the source ( $1 \mathrm{~mm}$ diameter) and the lambertian feature make it similar to a theoretical point source when observed with a standard lens. With the microscope lens, a simple but realistic modelisation of the source is still possible (a uniform lambertian penny-shaped source is supposed).

- The lambertian feature corresponds to the radiation behaviour of the high emissivity coatings generally used for IR thermography, contrary to an electronic source (IR LED or IR LASER).

- The possibility to perform lock-in acquisitions thanks to the periodicity of the source, which helps one to considerably reduce the external disturbances.

- The reflective plane does not attenuate the multiple reflections between lens and target.

The target is composed of a mirror polished 1-mm thick steel plate with a conical central hole acting as a diaphragm. On the outside (face exposed to the camera), the hole has a diameter of $1 \mathrm{~mm}$ and on the inside (face in front of the black body) about 3 $\mathrm{mm}$. A chopping disc, driven by a DC gear motor, is fixed to the inside face. The plate equipped with the chopping disc is mounted on the DCN1000 extended black body with four long screws allowing enough clearance for the chopping disc system. The whole setup is presented in Figure 4. The black body is fixed onto a manual 3-axis micro-positioning table. Table and camera are fixed on a laboratory table and targetcamera orientation is checked.

The black body regulates the differential temperature between its emitting area and a platinum probe. This probe is located in the front of the black body case and measures the ambient temperature or the target temperature if one is fixed to the case. There is a difference of temperature between the centre of the target and the case because of the low thermal conductivity of the target itself and at the target-case contact. The drift of this difference is quantified by thermography and its characteristic time is about $30 \mathrm{~s}$. The rotating period of the chopping disc has to be shorter so that lock-in measures are not affected by these drifts. Moreover it has to be longer than the camera's acquisition period (i.e. $0.01 \mathrm{~s}$ in the case of $100 \mathrm{fps}$ ) to avoid beat phenomenon. The chosen period is about $0.5 \mathrm{~s}$. The differential temperature between the target and the emitting area has to be the highest possible to enhance the detection of artefacts. Nonetheless the influence of saturated pixels will not be addressed in this paper. The chosen differential temperature leads to signals $30 \%$ lower than the saturation level.

Each test consists in acquiring a sequence of several thousands of images when the temperature of the black body is stabilised. Except the chopping disc that is on, the target is motionless in front of the camera and its hole appears in the frame. During 
the data processing the sequence is separated in two groups of images, depending on the state of the source. If the signal measured in the hole is higher (resp. lower) than its mean value, the current image belongs to the "source on" group (resp. the "source off" group). The time average of each group is computed before calculating their difference. This differential image is eventually divided by the amplitude of the source to obtain the Measured Differential Rationalized Radiation (latter called MDRR). In a uniform area $(100 \times 100$ pix $)$ of this image, the standard deviation is less than $0.02 \%$ of the contrast of the target because of the great number of images initially acquired.

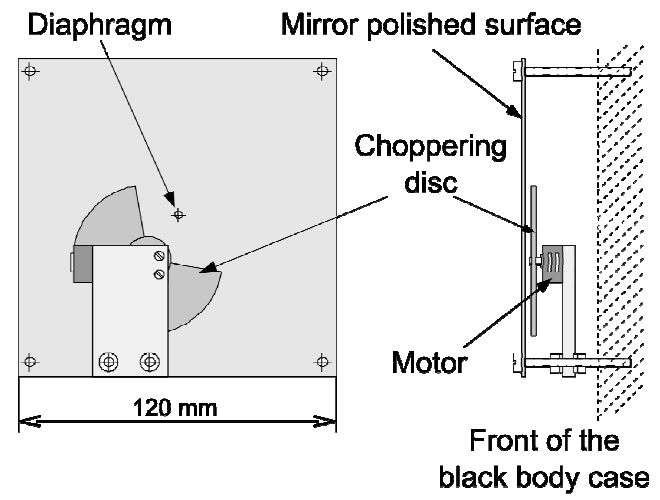

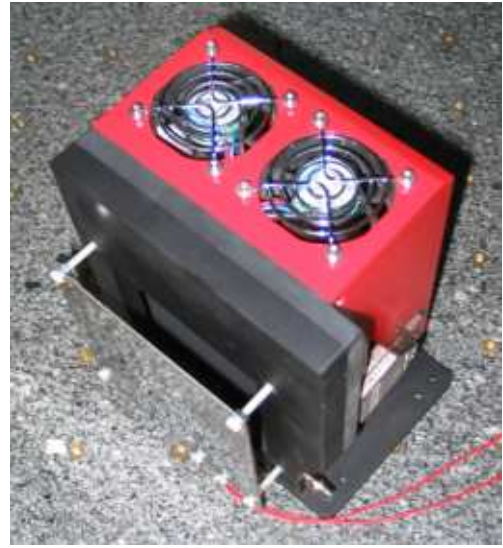

Figure 4. IR target: (a) principle of the target; (b) target fixed on the extended black body.

\subsection{Application}

One first uses the setup composed of the Jade III camera and the microscope lens and follows the experimental protocol described above. The target is placed at the nominal position of work (working distance $44.9+/-0.01 \mathrm{~mm}$, orientation: $90+/-0.1^{\circ}$ to the optical axis). Integration time is adequate for measurement at room temperature and the thermal contrast of the target is about $25 \mathrm{~K}$. The MDRR is presented In Figure 5.

The full scale image (Figure 5a) shows the position of source in the frame. When a smaller scale is used (Figure 5b), several unexpected heterogeneities are noticeable. Their maximum level is about $1 \%$ of the thermal contrast of the frame. They are of three different kinds:

- A positive halo, approximately symmetrical with respect to the optical axis. Its magnitude is about $0.6 \%$.

- A negative band appearing on the half of the image, oriented along the major axis of the frame. Its magnitude is about $-0.5 \%$. 

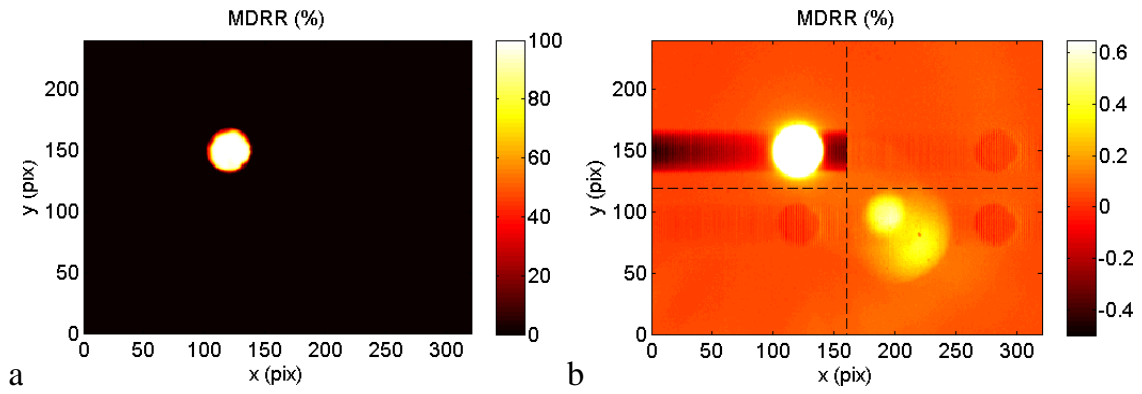

Figure 5. Measured Differential Rationalized Radiation (MDRR) for the Jade III camera and microscope lens. (a) Full scale ; (b) smaller scale to show low-level artefacts. Dashed lines delimit the $1 / 4$ frames of the sensor $(I T=1400 \mu \mathrm{s}, 150 \mathrm{fps}, 10000$ recorded images $)$.

- Three negative "ghost images", each in a different $1 / 4$ frame, of the same size as the source, whose positions follow a regularly spaced pattern oriented by the axis of the frame. Their magnitude is about $0.1 \%$.

The camera and lens are changed to deduce which artefact is linked to which component. The MDRR are presented in Figure 6 for Jade III with prime lens (a) and Titanium and microscope lens (b). In the first case, the positive halo is not present, but the two types of negative heterogeneities (band and "ghost images") are still there. Consequently the positive halo is supposed to be lens-related and the negative artefacts camera-related because only the lens has been changed between Figures $5 \mathrm{~b}$ and $6 \mathrm{a}$. The result for the third setup where only the camera has changed but not the lens (Figure 5b and 6b) confirms this hypothesis: one sees the positive halo but no longer the negative heterogeneities. Neither lens-related artefacts for the prime lens, nor camera-related artefacts for the Titanium camera are noticeable. The optical (lensrelated) artefact is thus studied with the Titanium camera with the microscope lens and the electronic ones with the Jade III and the prime lens.

The lens-related artefact clearly depends on the properties of the target surface: when a high emissivity coating covers it, the halo disappears. Consequently the heterogeneity is not only due to multiple reflections inside the lens (which correspond to usual "flare" effects), but also to reflections between the lens and the object. Even though the prime lens could also reflect part of the light back to the target, the high numerical aperture of the microscope lens $(N A=0.4)$ intensifies the phenomenon. When the target is translated in the range of the depth of field $[-0.1 \mathrm{~mm} 0.1 \mathrm{~mm}]$, the shape and intensity of the heterogeneity is unchanged. The heterogeneity is also independent of the tilt angle in the $\left[0^{\circ} 2^{\circ}\right]$ (above $2^{\circ}$ the left and right part of the target are out of the depth of field). The artefact is thus independent of the relative position of the lens to the target. As expected for a lens-related artefact, wherever is the source, the artefact is symmetrical with respect to the optical axis. When the source is taken away from the centre of the frame, shape and intensity evolve from sharp and intense to blurred and faded (Figure 7). Artefact intensity is in any case below $1 \%$ of the source intensity. 
Regarding the electronic artefacts, no variations with the frame rate (resp. with the integration time) were noticeable in the range [50 fps $150 \mathrm{fps}$ ] (resp. [140 $\mu \mathrm{s} 1400 \mu \mathrm{s}$ )). The variation with the source position is presented Figure 8 . The negative band is limited to the $1 / 4$ frame where the source is (Figures $8 \mathrm{a}-\mathrm{d}$ ). It is along the $x$ axis. It reappears at the left hand side of the same row (Figures $8,8 \mathrm{f}-\mathrm{g}$ ). The magnitude of the band decreases toward the positive $x$. This attenuation is independent of the $x$ position of the source and independent of the $1 / 4$ frame (Figures 8a-d). No matter in which $1 / 4$ frame the source is, a negative "ghost image" of the source appears in each of the other $3 \frac{1}{4}$ frames, along with positive bands of lower level (around 0,03\%). The distance between ghost images (or a ghost image and the source) along $x$ axis is equal to 160 pix, i.e. half the width of the frame. The distance from ghost images to the middle of the frame ( $y=120$ pix line) is equal to the distance from the source to the middle of the frame (Figures $8 \mathrm{a}, 8 \mathrm{e}-\mathrm{g}$ ). A close look at these electronic artefacts reveals that only one in two columns is affected (Figure 8h). The understanding and the modelisations of these radiometric artefacts will now be discussed.
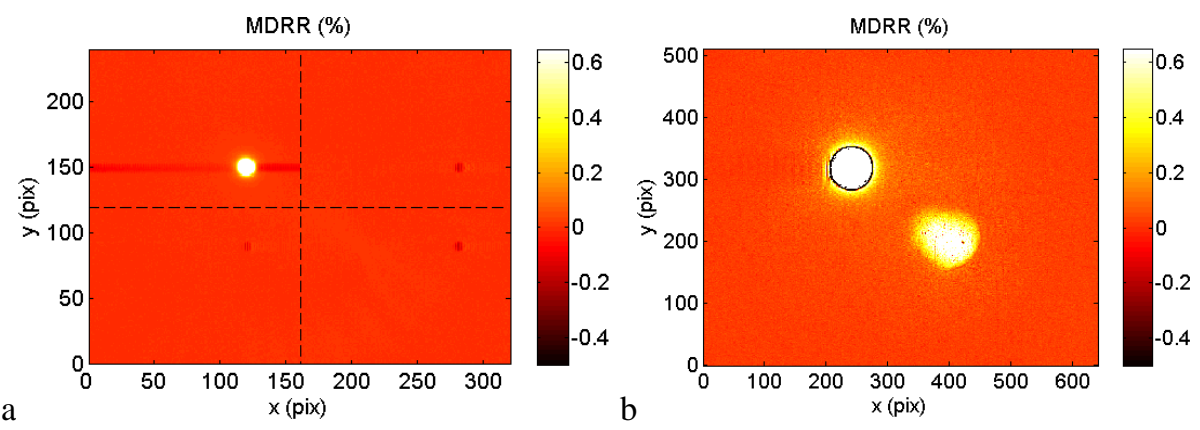

Figure 6. Measured Differential Rationalized Radiation (MDRR) for 2 different setups. (a) Jade III camera and prime lens. Dashed lines delimit the 1/4 frames of the sensor $(I T=1400 \mu \mathrm{s}, 150 \mathrm{fps}, 10000$ recorded images $) ;(b)$ Titanium camera and microscope lens (IT = $705 \mu \mathrm{s}, 52 \mathrm{fps}, 10000$ recorded images).

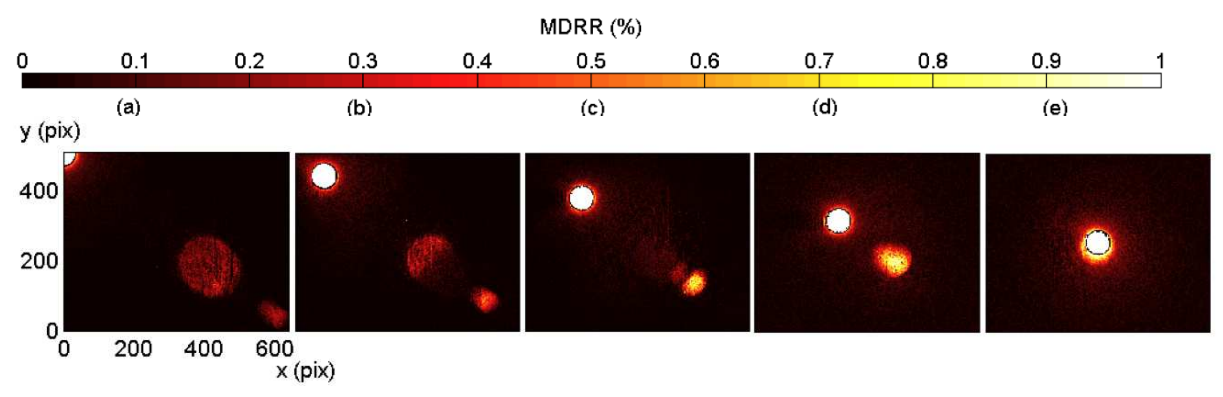

Figure 7. Measured Differential Rationalized Radiation (MDRR) for five different positions of the source (white disc). Titanium camera, microscope lens, IT =705 $\mu$ s, $52 \mathrm{fps}$. 


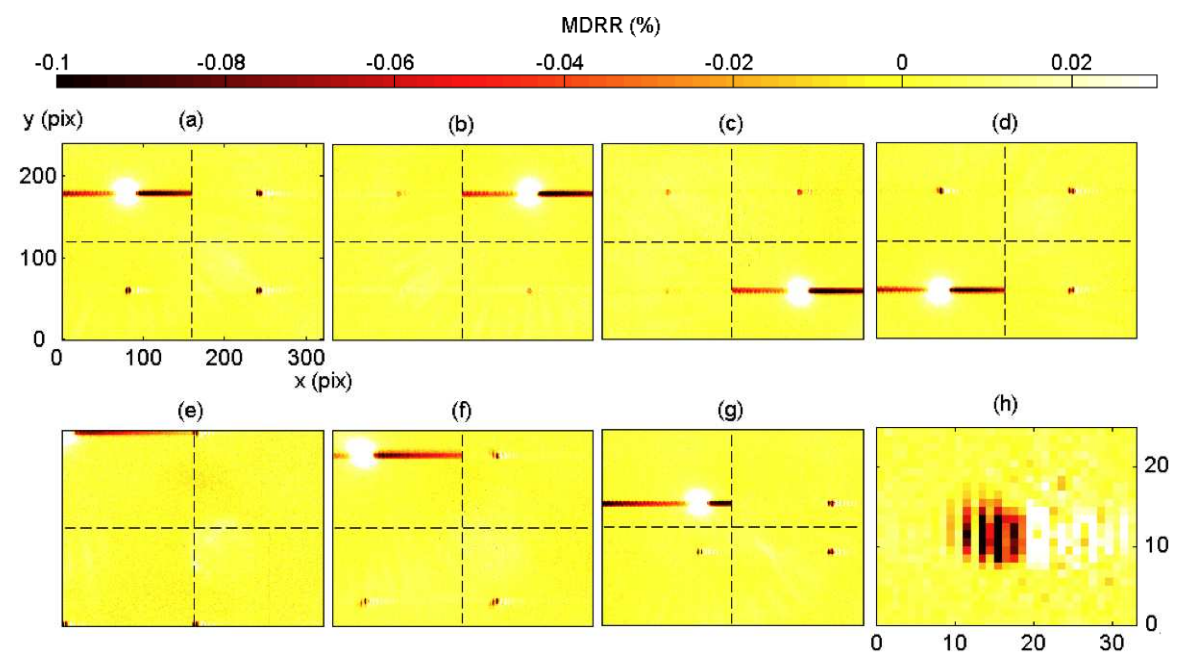

Figure 8. Measured Differential Rationalized Radiation (MDRR) for 7 positions of the source. Dashed lines delimit the $1 / 4$ frames of the sensor (Jade III camera, prime lens, $I T=1400 \mu$ s, $150 \mathrm{fps}$ ).

\section{Discussion}

A first attempt to simulate the contrast-induced optical artefact is made to improve its understanding. A numerical model is computed using the ray-tracing software Zemax $\odot$ (ZEMAX 2010). The microscope lens is completely modelled (i.e. mount and lenses with theoretical geometry and bulk materials properties). The reflection coefficient is set to a reasonable value of $1 \%$ for each thin-film coating. A 1-mm-diameter uniform pennyshaped IR source is placed in the object plane. The radiation of the source follows a $\cos ^{n}$ law with $n=5$ and a maximum radiation angle equal to $45.7^{\circ}$. Calculations use nonsequential mode. The maximum number of rays is set to $20 \times 10^{6}$. Figure 9 shows the intensity of radiation coming back to the object plane for two different positions of the source. These results are in good agreement with experimental observations:

- The magnitude of the reflected radiation is between $1 \%$ to $2 \%$ depending on the position of the source.

- The reflected radiation is symmetrical with respect to the optical axis. Moreover the reflection shape is identical to the source shape.

Nonetheless some differences exist. The optical artefact observed on the MDRR is slightly below $1 \%$. This value is raised if the reflection coefficient of the mirrorpolished target (about $90 \%$ ) is considered. Furthermore the measured shape is more blurred and depends on the position of the source (compare Figures 7 and 9). However, the origin of this optical artefact is confirmed. In reference to the Narcissus effect where the camera reflects on the object, this artefact is named "inverseNarcissus" effect because the source "reflects" on the lens. 


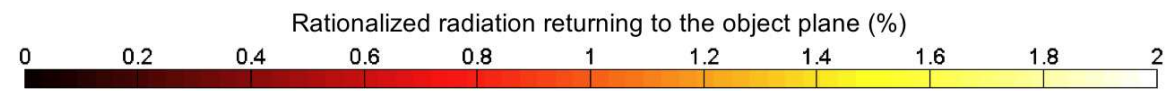

(a)

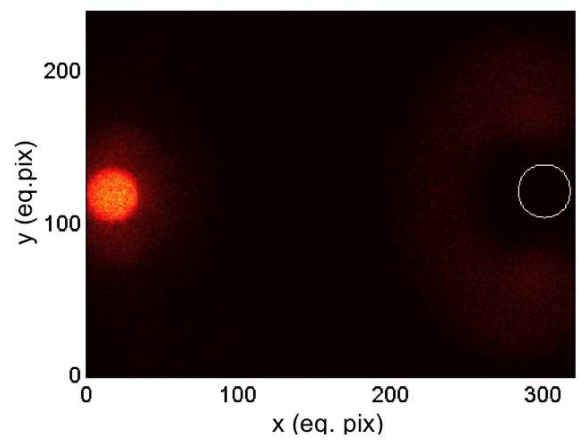

(b)

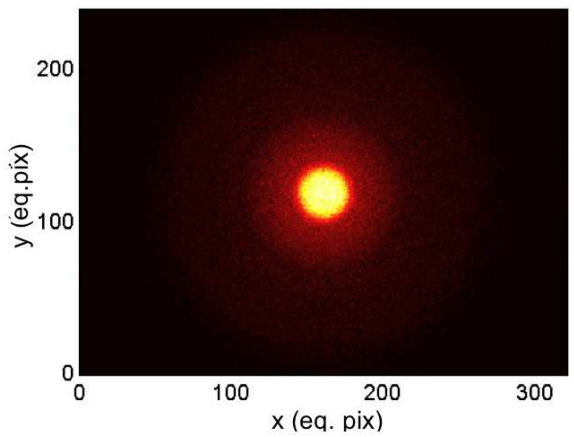

Figure 9. Numerical modelisation of the inverse Narcissus effect: Rationalized radiation returning to the object plane for (a) a off-centered disc source (white circle) and $(b)$ a centered disc source. Spatial unit is in "equivalent pixel" for microscope lens (1 pixel $=0.03 \mathrm{~mm}$ ).

Obviously, the simplest way to prevent this artefact is to use a high emissivity coating on the studied object. When the rough surface can not or must not be coated for other reasons, an other solution would be to correct this artefact a posteriori. In Eq. [2], the form of the correlation function $g$ has to be chosen and then fitted on raytracing calculations. This possibility is reasonable in the case of flat objects, since the artefact is not very dependent on the relative position of the lens.

Concerning the correction algorithm for the vignetting and Narcissus effects, it turns out to be efficient with only 6 parameters. As expected, the corrected images are still spoilt by the other artefacts, especially sensor non-uniformity. This remaining non-uniformity is the same whatever lens is corrected, which shows that the correction does not interfere with sensor own heterogeneity.

As for the "contrast-induced electronic artefacts" (ghost images and bands) an explanation of their origin is found in the way the ROC works. A study of Jade III raw images leads to the conclusion that its ROC follows a 4-channel multiplexed reading order type. This is in good agreement with the relative position of the negative "ghost images", the negative bands in each $1 / 4$ frame and the one-in-two feature. Although not yet precisely pinpointed, a "memory" effect of the embedded electronic data processing may be the only cause of these artefacts.

\section{Conclusion}

This work is part of a general study on the artefacts of IRFPA cameras and their corrections. In this paper, only the radiometric artefacts are investigated. The various 
origins of these artefacts have been briefly presented to facilitate test results interpretation. Two experimental protocols are proposed, the first one to detect and correct vignetting and Narcissus effects and the second one to assess contrast-induced artefacts. They are applied to two cameras and two lenses built by CEDIP-FLIR:

- The level of the heterogeneity due to vignetting and Narcissus effects is important for both lenses. The proposed correction method relies on the optical properties of such effects, so that it needs few parameters. It applies to the case of uniform emissivity. The corrected images no longer show halos, but only sensor pattern. The correction is thus only related to the lens and not to the rest of the camera.

- A special target was designed to detect contrast-induced artefacts. Two different artefacts were differentiable: optical ones and electronic ones.

- A particular optical artefact, called inverse Narcissus effect, appears only with the microscope lens. Its magnitude is less than $1 \%$ of the effective dynamic range. They are successfully modelled by ray-tracing. The best solution is to use a high emissivity coating to cover the object when possible, otherwise an a posteriori correction based on ray-tracing could be envisaged in the case of flat objects.

- Electronic artefacts are only detected on Jade III camera. Their magnitude is less than $1 \%$ of the frame contrast, so that they are not negligible in the case of low-level heterogeneities studies. The Read-Out-Circuit is responsible for them, so that raising surface emissivity will not solve this problem. Framing little contrasted images is the best solution, or an a posteriori correction has to be installed.

A post-processing correction of these artefacts is currently developed. It is composed of different "steps", each dedicated to one specific artefact. The correction of vignetting and Narcissus is the first completed step. The correction of the ROCrelated artefacts and the sensor non-uniformity artefacts are on their ways. The case of the geometrical aberrations will be investigated in a future article with the same sequential physics-based corrections framework.

\section{Acknowledgments}

The authors would like to thank Mr Vaché and Mr Brémond (Cedip-FLIR) respectively for their helpful work on the simulation of the inverse Narcissus effect and their technical support on Titanium camera. This paper greatly benefited from the GDR 2519 thermography workshops and the authors are very grateful for the contribution of their organisers MM. Grediac and Chrysochoos.

\section{References}

Bouferra R., Pron H., Henry J.-F., Bissieux C., Beaudoin J.L., Study of the intrinsic dissipation associated to the plastic work induced by a ball impact, International Journal of Thermal Sciences, 44 (2005) 115-119. 
Castelein P., Vision IR: du composant à l'image, Caractérisation et modélisation de composants matriciels infrarouge, C. R. Physique 4 (2003) 1167-1174.

CEDIP 2004 L0106.S01 Rev. B MW 50mm F2 Jade Lens, Lens Specification Sheet, Cedip Infrared Sytems (2004).

CEDIP 2005 L0120.S01 Rev. B MW Gx1 F2 Jade Lens, Lens Specification Sheet, Cedip Infrared Sytems (2005).

Chrysochoos A., Maisonneuve O., Martin G., Caumon H., Chezeaux J.C., Plastic and dissipated work and stored energy, Nuclear Engineering and Design, 114(3) (1989) 323-333.

Chrysochoos A., La thermographie infrarouge, un outil en puissance pour étudier le comportement des matériaux, Mécanique \& Industries, 3 (2002) 3-14.

Doudard C., Poncelet M., Calloch S., Boué C., Hild F., Galtier A., Determination of an HCF criterion by thermal measurements under biaxial cyclic loading, International Journal of Fatigue, 29(4) (2007), 748-757.

GUM 2008, Evaluation of measurement data - Guide to the expression of uncertainty in measurement, GUM 1995 with minor corrections, JCGM 100:2008.

Honorat V., Moreau S., Muracciole J.-M., Wattrisse B., Chrysochoos A., Calorimetric analysis of polymer behaviour using a pixel calibration of an IRFPA camera. QIRT Journal, 2 (2005) 153-172.

Kumar A., Sarkar S., Agarwal R.P., A novel algorithm and hardware implementation for correcting sensor non-uniformities in infrared focal plane array based staring system, Infrared Physics \& Technology 50 (2007) 9-13.

La Rosa G., Risitano A., Thermographic methodology for rapid determination of the fatigue limit of materials and mechanical components, Intern. J. of Fatigue, 22(1) (2000) 65-73.

Louche H., Vacher P., Arrieux R., Thermal observations associated with the Portevin-Le Châtelier effect in an Al-Mg alloy, Material Sci. and Engin.. A, 404 (2005), 188-196.

Pastor M.L., Balandraud X., Grédiac M., Robert J.L., Applying infrared thermography to study the heating of 2024-T3 aluminium specimens under fatigue loading, Infrared Physics \& Technology, 51(6) (2008) 505-515.

Poncelet M., Multiaxialité, hétérogénéités intrinsèques et structurales des essais d'autoéchauffement et de fatigue à grand nombre de cycles. PhD thesis, ENS Cachan (2007).

Poncelet M., Doudard C., Calloch S., Hild F., Weber B., Dissipation Measurements in Steel Sheets Under Cyclic Loading by Use of Infrared Microthermography, Strain, 42 (2010) 101-116.

Pron H., Bissieux C., Focal Plane Array infrared cameras as research tools, QIRT Journal, 1 (2004) 229-240.

Schulz M., Caldwell L., Non-uniformity correction and correctibility of focal plane arrays, Infrared Physics \& Technology 36 (1995) 763-767.

Shi Y., Zhang T., Zhigou C., Hui L., A feasible approach for non uniformity correction in IRFPA with nonlinear response, Infrared Physics \& Technology 46 (2005) 329-337.

ZEMAX 2010 : Software For Optical System Design. http://www.zemax.com/ 